\title{
The human papillomavirus vaccine: The promise of cervical cancer prevention
}

\author{
BL Johnston $M D^{1}$, JM Conly $M D^{2}$
}

$\mathrm{I}_{\mathrm{n}}^{\mathrm{n}}$

In February 2007, the National Advisory Committee on Immunization (NACI) - the expert committee that provides the Public Health Agency of Canada with advice relating to immunization - recommended that females between nine and 26 years of age be given the human papillomavirus (HPV) vaccine (1). Within one month, the Government of Canada announced in its federal budget that $\$ 300$ million would be allocated to the provinces for the HPV vaccine (2). Immunization recommendations are not infrequently associated with a certain amount of controversy, and HPV vaccination is no exception (3). A review of HPV disease and its diagnosis, as well as the evidence supporting the vaccination, will help to put the discussion into context.

Papillomaviruses are nonenveloped, double-stranded, circular DNA viruses. The structural integrity of the capsid proteins is necessary to elicit protective antibodies and has been an important consideration in vaccine development (4). There are two capsid proteins, L1 (major) and L2 (minor), against which neutralizing antibodies may be formed (4). Approximately 100 different types of HPVs have been characterized molecularly (5). They are host specific, and each type is characteristically associated with a distinct histopathological process, ranging from genital and nongenital warts to various forms of invasive cancer (6). The L2 protein is located internally within the capsid. While the anti-L2 antibodies appear to show some cross-reactivity to heterologous HPV types, they are less potent than the anti-L1 antibodies, which appear to be type specific (4).

HPVs infect primitive (undifferentiated) basal keratinocytes, within which they replicate without causing cell damage or death (7). There is no strong signal to the host that a foreign antigen is present, thus allowing the HPV to evade the host immune system for long periods of time. Oncogenic strains are capable of integrating into the host cell genome, leading to persistent infection and potential for malignancy (8). HPV tends to cause cancer at areas known as transformation zones, in which one kind of epithelium contacts and gradually replaces another, explaining its predilection to cause cervical, anal and oropharyngeal cancers (8). Ultimately, however, an immune response is generated in most instances. Animal experiments and natural history studies in humans indicate that this is characterized by a strong local cellmediated response, followed by the appearance of a serum neutralizing antibody that is protective against reinfection (7). The observed median time to clearance of prevalent infections in various studies has ranged from between four and six months to between one and two years (8). Viral clearance is not often followed by reappearance of the same genotype (8).

Epidemiological studies (9), supported by molecular technology, have provided evidence of the causal role of some HPV types in the development of cervical cancer. Indeed, it has been claimed that HPV represents the first necessary cause of a human cancer. In epidemiological terms, necessary means that the cancer will only develop if the agent is present. Pooled data from 11 case-control studies (10) from nine countries have identified 15 HPV types (types 16, 18, 31, 33, 35, 39, 45, 51, 52, 56, 58, 59, 68, 73 and 82) as carcinogenic (high-risk), and three additional HPV types (types 26, 53 and 66) as probably carcinogenic. HPV-16 and HPV-18 account for $70 \%$ of cervical cancers (11). While the type distribution varies somewhat geographically and according to histological type, a vaccine containing the seven most common HPV types (types 16, 18, 45, 31, 52, 33 and 58) would prevent approximately $87 \%$ of cervical cancer worldwide with little regional variation (12). Whether such a vaccine would be financially feasible and amenable to evaluation through randomized clinical trials (because of small numbers of cancer associated with non-HPV-16 and non-HPV-18 types) is unknown (12). HPV-16 appears to be the most carcinogenic of the HPVs, with an absolute risk of high-grade intraepithelial neoplasia approaching $40 \%$ at five years persistence (8). Despite the prevalence of high-risk HPV types, very few individuals develop premalignant or malignant anogenital disease (5). Apart from smoking, it has been difficult to identify risk factors for invasion that are independent of risks for HPV infection itself (8).

The age-standardized incidence rate for cervical cancer in Canadian females was approximately three of 100,000 females in 2004, down from 15 of 100,000 females in 1978 (13), a reduction that can be attributed largely to cervical cancer screening programs. This compares to approximately 100 of 100,000 females for breast cancer and 40 of 100,000 females for each of colorectal and lung cancers, placing cervical cancer as the sixth most common malignancy in women (13). It has

\footnotetext{
${ }^{1}$ Queen Elizabeth II Health Sciences Centre and Dalhousie University, Halifax, Nova Scotia; ${ }^{2}$ Departments of Medicine, Pathology and

Laboratory Medicine, and Microbiology and Infectious Diseases, University of Calgary, Calgary, Alberta

Correspondence and reprints: Dr BL Johnston, Queen Elizabeth II Health Sciences Centre, 5014ACC - 1278 Tower Road, Halifax,

Nova Scotia B3H 2Y9. Telephone 902-473-5553, fax 902-473-7394, e-mail ljohnsto@dal.ca

Received and accepted for publication July 27, 2007
} 
been estimated that there will be 377 deaths from cervical cancer in Canadian women in 2007, and 1400 new cases. This compares with 33,000 new cases and 14,000 deaths from lung and breast cancer in Canadian women in 2007. Worldwide, cervical cancer kills an estimated 250,000 women every year, with more than $80 \%$ of the 500,000 new cases occurring in the developing world, where cervical cancer is the foremost cause of cancer-related mortality in women (14).

HPV has been associated with a number of other cancers, although the strength of the association is generally not as well established as it is for cervical cancer. These cancers include anogenital and oropharyngeal malignancies (15). Estimates of the attributable fraction of cancer accounted for by HPV, however, demonstrate quite different etiological burdens presented by the virus for the various malignancies. Whereas HPV is responsible for all cervical cancers and $90 \%$ of anal cancers in both men and women, it has been estimated to be responsible for only $3 \%$ of oral cavity and $12 \%$ of oropharyngeal cancers (15). In Canada, it has been estimated that 3200 individuals are diagnosed each year with oral cancer of whom 1100 die (13). By extrapolation, there will be 133 HPV-related oropharyngeal cancer deaths in Canadian men and women in 2007, a cancer for which there is no screening program. Overall, by virtue of its causal role in cervical cancer, HPV is responsible for $5.2 \%$ of the world cancer burden $-2.2 \%$ in developed countries and $7.7 \%$ in developing countries (15).

Obviously, preventing HPV infection would decrease the cancer burden worldwide, with the greatest potential for benefit being in the developed world, and so developing a vaccine against HPV makes inherent good sense. HPV vaccines are preparations of L1 that, when expressed in yeast, assemble into virus-like particles that are structurally very similar to the natural virus. Preclinical experiments revealed that the recombinant virus-like particles were highly immunogenic when combined with an adjuvant (14), and observational studies over the years have suggested that neutralizing antibodies would be protective against new infections (7).

A number of randomized, placebo-controlled trials (16-21) evaluating the safety and efficacy of recombinant HPV vaccine have been published. The majority of these studies have included females between 15 and 26 years of age with few prior sexual partners (six partners or less). The participants may or may not have had prevalent and/or prior HPV infection. Several different outcome measures have been examined, including incident and prevalent HPV infection, the presence of genital warts or cancer, and histological abnormalities predictive of HPV disease. These histological abnormalities include cervical adenocarcinoma in situ, cervical intraepithelial neoplasia (CIN) grades 1 to 3, vulvar intraepithelial neoplasia grades 2 and 3, and vaginal intraepithelial neoplasia grades 2 and 3. Given the long latency period between infection and the appearance of cancer, and the need to treat premalignant lesions (rather than wait for them to evolve into an invasive cancer), surrogate markers of malignancy such as precancerous histological abnormalities have represented an important outcome measure in the clinical trials (4). Grade $1 \mathrm{CIN}$ is not considered to be precancerous, and up to $40 \%$ of grade 2 CIN lesions regress spontaneously (22). Grade $3 \mathrm{CIN}$ and adenocarcinoma in situ are the most predictive surrogate markers for invasive cancer potential (22). Studies $(16-18,23)$ have also evaluated the antibody response to immunization, acknowledging that the antibody correlates of protection are unknown.
Quadrivalent (HPV types 6, 11, 16 and 18) and bivalent (HPV types 16 and 18) recombinant vaccines have been studied. HPV types 6 and 11 are responsible for more than $90 \%$ of genital wart disease (23). While genital warts are not a cancer precursor, they are the most common of the viral sexually transmitted diseases and place an unquantified burden on the health care system. The quadrivalent vaccine is currently the only one licensed in Canada; licensing of the bivalent product is expected later in 2007. Both the quadrivalent and bivalent vaccines are administered as part of a three-dose series over six months.

In the randomized, controlled clinical trials, two types of analyses have generally been performed. The intention-totreat (ITT) analysis evaluates outcomes in females with incident or prevalent HPV infection during the vaccination period. The per-protocol analysis (PPA) examines females with no prior HPV infection who remain infection free throughout the first seven months of the trial and have no protocol violations. The ITT situation is thought to reflect the real-life scenario in which sexually active women and girls are exposed to HPV while undergoing immunization, whereas the PPA situation reflects the real-life scenario in which the girls and women have completed the vaccine series before exposure. The results of the ITT analyses allow some sense of vaccine effectiveness, whereas the PPA purely measures vaccine efficacy.

The results of the trials (24), some providing data up to five years following immunization, have been well publicized. While results vary according to the type of analysis performed and outcomes examined, both the bivalent and quadrivalent vaccines have had impressive efficacy (Table 1). In general, the vaccine has been more effective in preventing disease due to HPV-16 than HPV-18, and for protecting against the development of lower grade lesions. These studies are still of insufficient duration to fully quantify the benefit in terms of preventing cancer due to HPV. The benefit was also clearly lower in those who are exposed to HPV before completing the series (ITT) than in those who completed the series before acquiring HPV infection (PPA). The vaccine has been well tolerated in all studies to date (16-21). Whether postmarketing studies or longer-term follow-up will identify unpredicted adverse effects remains to be seen. When serum antibody levels have been measured, neutralizing antibodies remain elevated (17-19,23). In fact, antibody levels tend to be higher in those with vaccine-related immunity than in those with natural immunity $(17,18)$. It is still unknown, however, exactly how long the vaccine is protective in the absence of booster doses. Certainly, for many individuals, exposure to HPV remains a long-term, if not lifelong, risk.

Based on these findings, NACI made its recommendation to immunize females between nine and 26 years of age. The rationale for the younger age is to immunize girls before they become sexually active and are exposed to HPV. Although efficacy trials have not been conducted in this young age group for legal, logistical and ethical reasons, studies looking at the immune response to HPV vaccine in boys and girls between 10 and 15 years of age show higher antibody levels than in females between 16 and 23 years of age (23). Such a 'bridging efficacy' study supports the assumption that the vaccine is at least as effective in younger adolescents as in the clinical trial participants. The finding that $20 \%$ of 16 - to 23 -year-old participants in this study were HPV positive at baseline speaks to the need for 
TABLE 1

Summary of vaccine efficacy $(\%[95 \% \mathrm{Cl}])$ in selected human papillomavirus vaccine clinical trials

\begin{tabular}{|c|c|c|c|c|c|c|c|}
\hline \multirow[b]{2}{*}{$\begin{array}{l}\text { Vaccine } \\
\text { (reference) }\end{array}$} & \multirow[b]{2}{*}{$\mathbf{n}$} & \multicolumn{5}{|c|}{ Outcome } & \multirow[b]{2}{*}{ ASCUS } \\
\hline & & Analyses & Persistent infection & Incident infection & $\mathrm{CIN}-1$ & $\geq \mathrm{CIN}-2$ and $\mathrm{CIN}-3$ & \\
\hline \multirow[t]{2}{*}{ Quad (16) } & $266 \mathrm{v}$ & PPA & $95.6(83.8-99.5)$ & N/A & - & $100(<0.0-100)$ & N/A \\
\hline & $263 p$ & ITT & $93.5(82.5-98.3)$ & $\mathrm{N} / \mathrm{A}$ & - & $100(30.8-100)$ & $\mathrm{N} / \mathrm{A}$ \\
\hline \multirow[t]{2}{*}{ Bival (17) } & $393 v$ & PPA & $96.0(75.2-99.9)$ & $94.7(83.5-98.9)$ & & & \\
\hline & $383 p$ & ITT & $94.4(78.2-99.4)$ & $88.5(77.0-95.0)$ & $100(42.4-100)$ & $100(-7.7-100)$ & $95.7(83.5-99.5)$ \\
\hline \multirow[t]{2}{*}{ Bival (18) } & $560 \mathrm{v}$ & PPA & $100(76.8-100)$ & $73.6(49.7-86.1)$ & - & $\mathrm{N} / \mathrm{A}$ & $\mathrm{N} / \mathrm{A}$ \\
\hline & $553 p$ & ITT & $87.5(64.6-95.6)$ & $67.6(48.9-79.4)$ & - & $\mathrm{N} / \mathrm{A}$ & $92.9(70.0-98.3)$ \\
\hline \multirow[t]{2}{*}{ Quad (19) } & 277 v & PPA & $89(70-97)$ & $\mathrm{N} / \mathrm{A}$ & - & $\mathrm{N} / \mathrm{A}$ & $\mathrm{N} / \mathrm{A}$ \\
\hline & $275 p$ & ITT & $88(72-96)$ & N/A & - & $100(32-100)$ & N/A \\
\hline \multirow[t]{2}{*}{ Quad (20) } & $5305 v$ & PPA & N/A & $\mathrm{N} / \mathrm{A}$ & - & $98(86-100)$ & $\mathrm{N} / \mathrm{A}$ \\
\hline & $5260 p$ & ITT & $\mathrm{N} / \mathrm{A}$ & N/A & - & $44(26-58)$ & $\mathrm{N} / \mathrm{A}$ \\
\hline \multirow[t]{2}{*}{ Quad (21) } & 2723 v & PPA & $\mathrm{N} / \mathrm{A}$ & $\mathrm{N} / \mathrm{A}$ & $100(92-100)$ & CIN-2: 100 (81-100), CIN-3: 100 (76-100) & $\mathrm{N} / \mathrm{A}$ \\
\hline & $2732 p$ & ITT & N/A & N/A & $62(46-74)$ & CIN-2: $30(<0-56)$, CIN-3: $12(<0-44)$ & N/A \\
\hline
\end{tabular}

ASCUS Atypical squamous cells of undetermined significance; Bival Bivalent; CIN Cervical intraepithelial neoplasia; ITT Intention-to-treat; N/A Not applicable; p Placebo; PPA Per-protocol analysis; Quad Quadrivalent; v Vaccine

introducing the vaccine in the early teenage years or even earlier. In their summary statement (1), NACI cited the 2002 Canadian Youth, Sexual Health and HIV/AIDS Study finding that $19 \%$ of grade 9 girls reported having had vaginal intercourse at least once. Once they become sexually active, approximately $50 \%$ of girls and women acquire HPV infection within three years (5).

Despite the data above, a number of questions remain. How long will protection last? Will boosters be required? Exactly how effective will the vaccine be? Does it make sense to target only one sex in prevention strategies against a sexually transmitted infection? If the goal is cervical cancer prevention, modeling has suggested that immunizing boys would affect its incidence only marginally (4). However, Garnett (25) has argued that the benefit of also immunizing boys could be greater if vaccine uptake in girls is low. Additionally, if oropharyngeal cancer rates continue to increase, with the recognition that it too may be an HPV-related sexually transmitted disease (26), arguments may be made to broaden the vaccine recommendation to include boys. While Canada does not seem to have experienced the political debate observed in the United States around HPV vaccination $(3,27)$, it is unclear whether that will remain the case as vaccine programs roll out across the country. Finally, will HPV-16 and HPV-18 be replaced by other cancer-causing types (22)?

Will this be a cost-effective vaccine? The current approach toward cervical cancer prevention is Papanicolaou cytological screening followed by triage of cases with abnormal cytology for management of cancerous and precancerous lesions (28). This will not end with the HPV vaccine. First, there will remain, for a number of years to come, a cohort of unvaccinated women. Second, the vaccine does not cover $20 \%$ to $30 \%$ of cancer-causing types. Incidentally, it is not clear how reduced cervical cancer incidence will alter the performance characteristics of the test, because technologists examine fewer specimens and even fewer abnormal specimens (28). In their modeling analysis of the potential cost-effectiveness of prophylactic HPV vaccines in Canada, Brisson et al (29) estimated that vaccinating 12-year-old girls (assuming 95\% efficacy and $\$ 400$ cost/course) against HPV-16 and HPV-18, or HPV-6, HPV-11, HPV-16 and HPV-18 would cost $\$ 31,000$
(95\% CI $\$ 15,000$ to $\$ 55,000)$ and $\$ 21,000$ (95\% CI $\$ 11,000$ to $\$ 30,000$ ) per quality-adjusted life-years gained, respectively. They concluded that unless screening is modified, the treatment costs saved through immunization are insignificant compared with the cost of such a program. It rapidly becomes apparent that this cost is well beyond what developing countries, those most likely to benefit from HPV vaccination, can afford, given vaccine costs in the developed world.

It is difficult to argue against a vaccine that holds so much promise in the prevention of cancer. However, there are many valuable initiatives that warrant consideration in a publicly funded system. Erickson et al (30) have derived an analytical framework for immunization programs in Canada. In addition to the categories of disease burden and vaccine characteristics, they recommend consideration of a number of other criteria broadly covering cost-effectiveness, acceptability, feasibility, program evaluability, research questions, equity, ethical, legal and political considerations. NACI has given consideration to disease burden and vaccine characteristics, and has made its recommendation (1). The Canadian Immunization Committee may have a challenging task ahead because it has to examine the other criteria that need to be addressed when considering a national HPV immunization program in Canada.

\section{REFERENCES}

1. National Advisory Committee on Immunization. Statement on human papillomavirus vaccine. An Advisory Committee Statement (ACS). Can Commun Dis Rep 2007;33:1-32.

2. André Picard. Scientific breakthrough or unproven fix? The Globe and Mail 2007; March 27: A17.

3. Comeau P. Debate begins over public funding for HPV vaccine. CMAJ 2007;176:913-4. (Errata in 2007;176:1310, 2007;177:66).

4. Arbyn M, Dillner J. Review of current knowledge on HPV vaccination: An appendix to the European Guidelines for Quality Assurance in Cervical Cancer Screening. J Clin Virol 2007;38:189-97.

5. Frazer IH, Cox JT, Mayeaux EJ, et al. Advances in prevention of cervical cancer and other human papillomavirus-related diseases. Pediatr Infect Dis J 2006;25(2 Suppl):S65-82.

6. Bonnez W, Reichman RC. Papillomaviruses. In: Mandell GL, Bennett JE, Dolin R, eds. Principles and Practice of Infectious Diseases, 6th edn. Philadelphia: Elsevier Churchill Livingstone, 2005:1841-56.

7. Stanley M. Immune responses to human papillomavirus. Vaccine 2006;24(Suppl 1):S16-22. 
8. Moscicki AB, Schiffman M, Kjaer S, Villa LL. Chapter 5: Updating the natural history of HPV and anogenital cancer. Vaccine 2006;24(Suppl 3):S42-51.

9. Bosch FX, Lorincz A, Muñoz N, Meijer CJLM, Shah KV. The causal relation between human papillomavirus and cervical cancer. J Clin Pathol 2002;55:244-65.

10. Muñoz N, Bosch FX, de Sanjosé S, et al; International Agency for Research on Cancer Multicenter Cervical Cancer Study Group. Epidemiologic classification of human papillomavirus types associated with cervical cancer. N Engl J Med 2003;348:518-27.

11. Clifford G, Franceschi S, Diaz M, Muñoz N, Villa LI. Chapter 3: HPV type-distribution in women with and without cervical neoplastic diseases. Vaccine 2006;24(Suppl 3):S26-34.

12. Muñoz N, Bosch FX, Castellsagué X, et al. Against which human papillomavirus types shall we vaccinate and screen? The international perspective. Int J Cancer 2004;111:278-85.

13. http://www.cancer.ca/vgn/images/portal/cit_86751114/36/15/ 1816216925cw_2007stats_en.pdf (Version current as July 11, 2007).

14. Pagliusi SR, Teresa Aguado M. Efficacy and other milestones for human papillomavirus vaccine introduction. Vaccine 2004;23:569-78.

15. Parkin DM. The global health burden of infection-associated cancers in the year 2002. Int J Cancer 2006;118:3030-44.

16. Villa LL, Costa RL, Petta CA, et al. High sustained efficacy of a prophylactic quadrivalent human papillomavirus types 6/11/16/18 L1 virus-like particle vaccine through 5 years of follow-up. Br J Cancer 2006;95:1459-66.

17. Harper DM, Franco EL, Wheeler CM, et al; HPV Vaccine Study group. Sustained efficacy up to 4.5 years of a bivalent L1 virus-like particle vaccine against human papillomavirus types 16 and 18: Follow-up from a randomised control trial. Lancet 2006;367:1247-55.

18. Harper DM, Franco EL, Wheeler C, et al; GlaxoSmithKline HPV Vaccine Study Group. Efficacy of a bivalent L1 virus-like particle vaccine in prevention of infection with human papillomavirus types 16 and 18 in young women: A randomised controlled trial. Lancet 2004;364:1757-65.

19. Villa LL, Costa RL, Petta CA, et al. Prophylactic quadrivalent human papillomavirus (types 6,11,16, and 18) L1 virus-like particle vaccine in young women: A randomised double-blind placebo-controlled multicentre phase II efficacy trial. Lancet Oncol 2005;6:271-8.

20. FUTURE II Study Group. Quadrivalent vaccine against human papillomavirus to prevent high-grade cervical lesions. N Engl J Med 2007;356:1915-27.

21. Garland SM, Hernandez-Avila M, Wheeler CM, et al; Females United to Unilaterally Reduce Endo/Ectocervical Disease (FUTURE) I Investigators. Quadrivalent vaccine against human papillomavirus to prevent anogenital diseases. N Engl J Med 2007;356:1928-43.

22. Sawaya GF, Smith-McCune K. HPV vaccination - more answers, more questions. N Engl J Med 2007;356:1991-3.

23. Neame D. Make HPV vaccination a priority in your practice. The Medical Post 2007;43:11.

24. Block SL, Nolan T, Sattler C, et al; Protocol 016 Study Group. Comparison of the immunogenicity and reactogenicity of a prophylactic quadrivalent human papillomavirus (types 6, 11, 16, and 18) L1 virus-like particle vaccine in male and female adolescents and young adult women. Pediatrics 2006;118:2135-45

25. Garnett GP. Role of herd immunity in determining the effect of vaccines against sexually transmitted disease. J Infect Dis 2005;191(Suppl 1):S97-106.

26. D'Souza G, Kreimer AR, Viscidi R, et al. Case-control study of human papillomavirus and oropharyngeal cancer. N Engl J Med 2007; 356:1944-56

27. Franco EL, Cuzick J, Hildesheim A, de Sanjosé S. Chapter 20: Issues in planning cervical cancer screening in the era of HPV vaccination. Vaccine 2006;24(Suppl 3):S171-7.

28. Charo RA. Politics, parents, and prophylaxis - mandating HPV vaccination in the United States. N Engl J Med 2007;356:1905-8

29. Brisson M, Van de Velde N, De Wals P, Boily MC. The potential cost-effectiveness of prophylactic human papillomavirus vaccines in Canada. Vaccine 2007;25:5399-408.

30. Erickson LJ, DeWals P, Farand L. An analytical framework for immunization programs in Canada. Vaccine 2005;23:2470-6. 


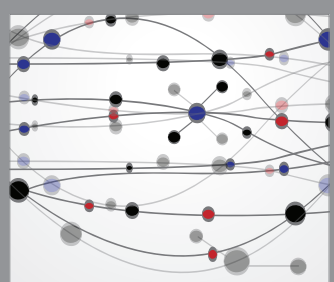

The Scientific World Journal
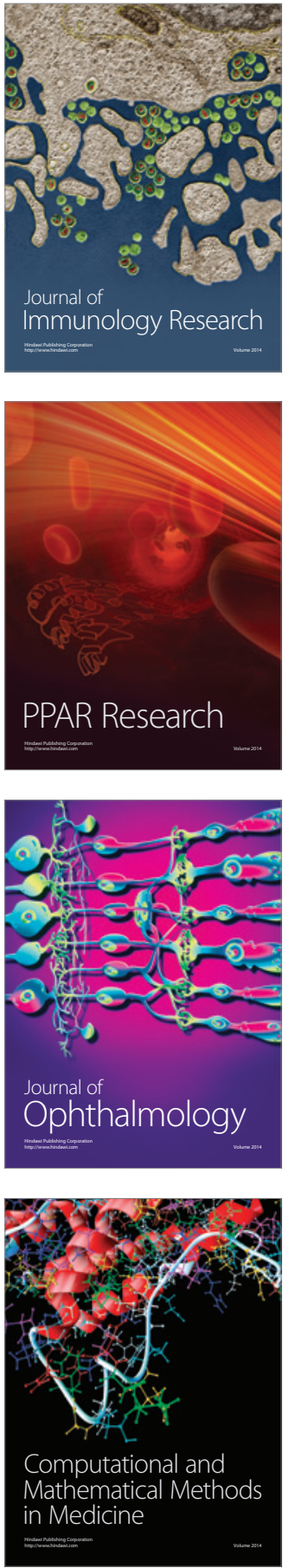

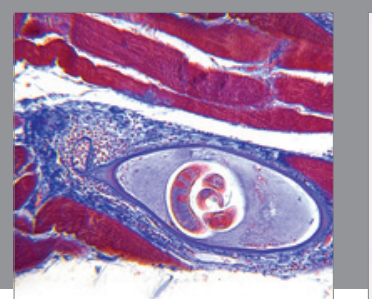

Gastroenterology Research and Practice

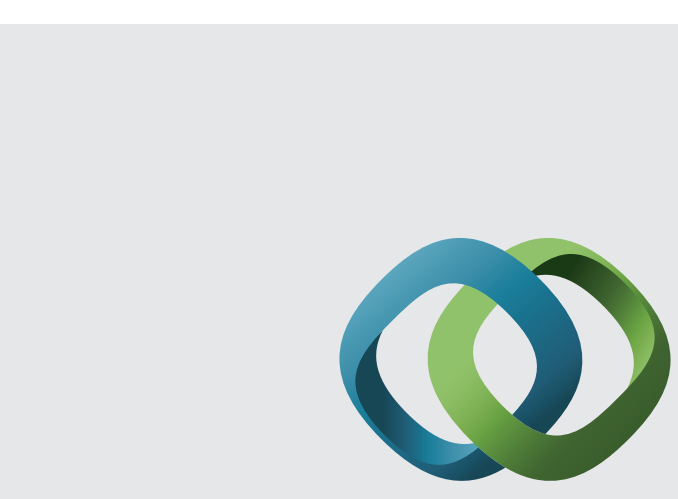

\section{Hindawi}

Submit your manuscripts at

http://www.hindawi.com
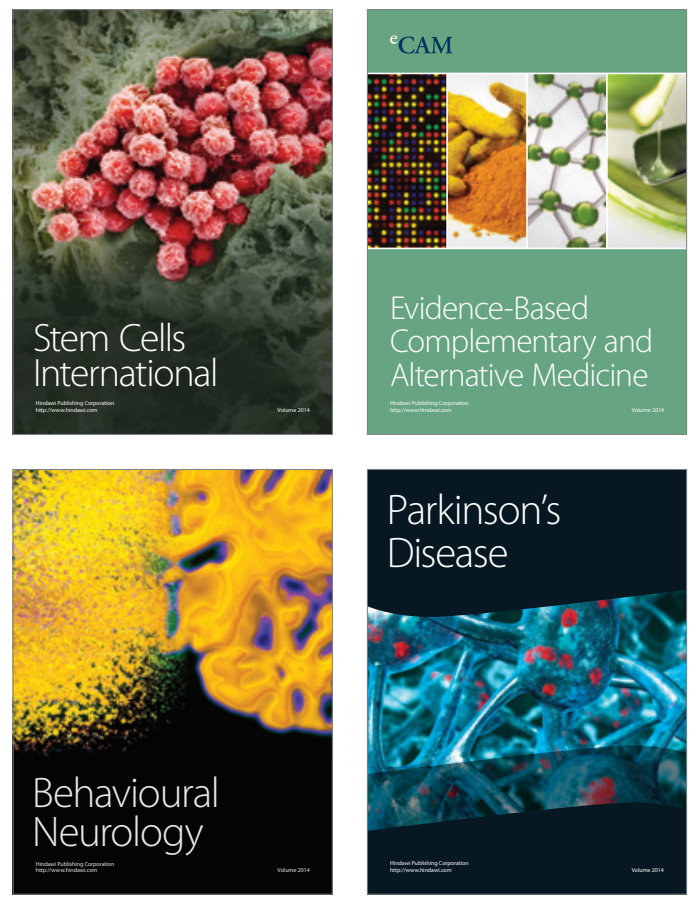
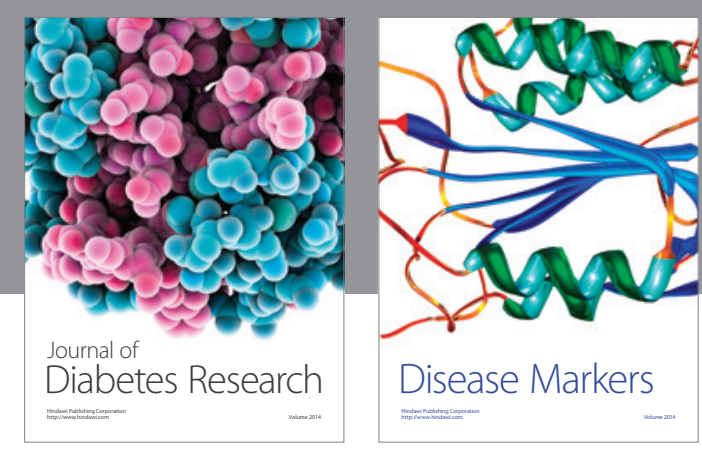

Disease Markers
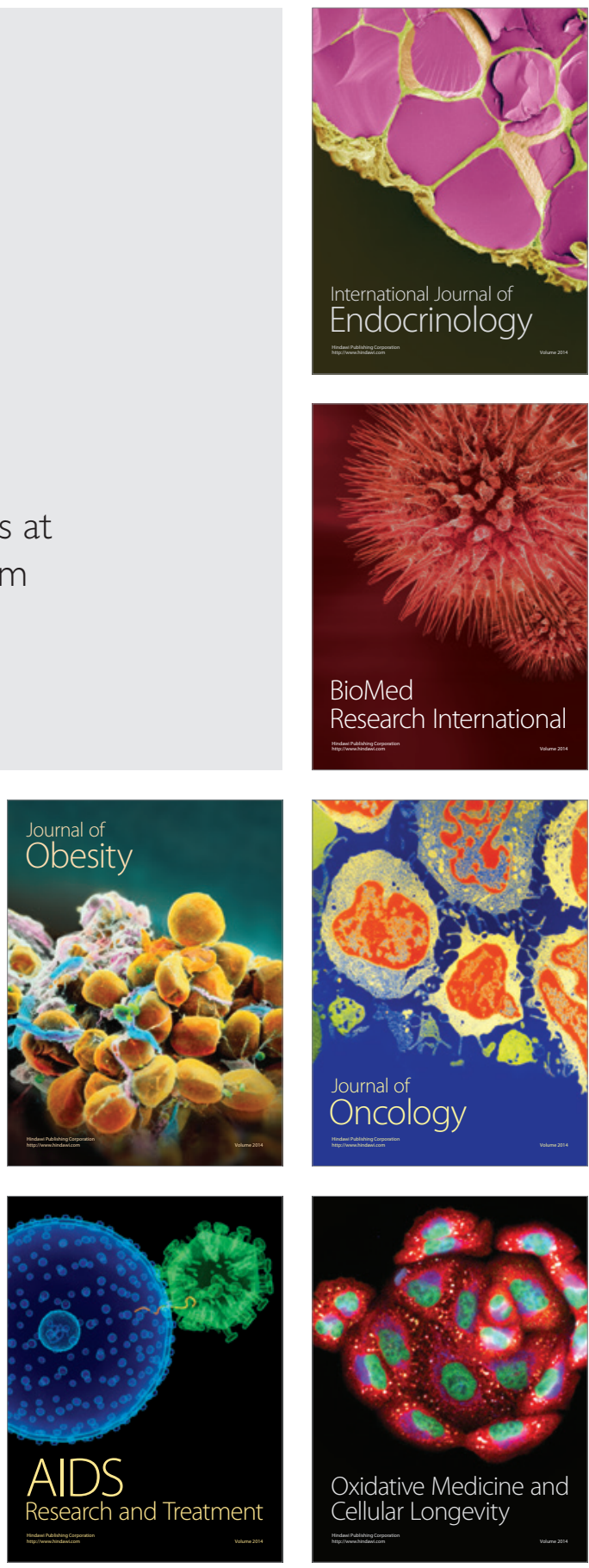